J. Austral. Math. Soc. (Series A) 43 (1987), 74-80

\title{
SUPPORT OF THE JOINT SPECTRAL MEASURE
}

\author{
JAN NIECHWIEJ \\ (Received 28 January 1986, revised 22 April 1986) \\ Communicated by R. O. Vyborny
}

\begin{abstract}
For a doubly commuting $n$-tuple of unbounded normal operators in a Hilbert space the joint spectral measure can be constructed and its closed support described as the joint spectrum of the given $n$-tuple. The same is here shown for larger, possibly uncountable, families of operators.
\end{abstract}

1980 Mathematics subject classification (Amer. Math. Soc.): 47 A 10, 47 B 15; Secondary 46 L 99.

\section{Introduction}

A. B. Patel in [3] has established the joint spectral theorem for a doubly commuting $n$-tuple of unbounded normal operators. What seems to be most interesting in Patel's result, apart from a new technique, is an explicit formula for the closed support of the constructed joint spectral measure, characterizing it as the joint spectrum of the given $n$-tuple of operators.

The existence of the joint spectral measure for an arbitrary, even uncountable, family of normal operators whose resolutions of the identity commute is also known (see [1] or [2]), but without such an algebraic characterization of its support. In what follows it is shown that doubly commuting normal operators have commuting resolutions of the identity. Accordingly, the joint spectral measure exists [1]. It turns out that the support of the joint spectral measure can be characterized as the joint spectrum of the given family of operators. Thus a

(C) 1987 Australian Mathematical Society $0263-6115 / 87 \$ A 2.00+0.00$ 
version of the result from [3] is valid for any family of normal doubly commuting operators, although the method used there cannot be applied when the family of normal operators is too numerous.

\section{Introductory definitions}

Let $J$ be a set of indices and $\left(T_{j}\right)_{j \in J}$ be a family of normal (possibly unbounded) operators acting in a Hilbert space $H$.

1.1. Definition ([3]). The family $\left(T_{j}\right)_{j \in J}$ is said to be doubly commuting iff for any $i, j \in J$ there is $T_{i} T_{j}=T_{j} T_{i}$ and $T_{i} T_{j}^{*}=T_{j}^{*} T_{i}$.

The above equalities are in the usual sense of equality of unbounded operators. That is, if $D(A)$ denotes the domain of an operator $A$, then $D\left(T_{i} T_{j}\right)=D\left(T_{j} T_{i}\right)$ and for any $x \in D\left(T_{i} T_{j}\right)$ there is $T_{i} T_{j} x=T_{j} T_{i} x$ and similarly the domains of the operators $T_{i} T_{j}^{*}$ and $T_{j}^{*} T_{i}$ are equal and the values of these operators are the same.

Now let $\mathbf{T}=\left(T_{j}\right)_{j \in J}$ be a family of normal operators.

1.2. Definition. An element $\left(\lambda_{j}\right)_{j \in J}$ from $\mathbf{C}^{J}$ belongs to the joint resolvent set $\rho(\mathbf{T})$ if there exist a natural number $N$, indices $j_{1}, j_{2}, \ldots, j_{n} \in J$ and bounded linear operators $B_{1}, B_{2}, \ldots, B_{N}$ acting in $H$ such that $\sum_{n=1}^{N} B_{n}\left(T_{j_{n}}-\lambda_{j_{n}} I\right) \subset I$, where $I$ is the identity operator. The joint spectrum of the family $\mathbf{T}=\left(T_{j}\right)_{j \in J}$ is the set $\operatorname{Sp}(\mathbf{T})=\mathbf{C}^{J} \backslash \rho(\mathbf{T})$.

When one considers a $n$-tuple of normal operators (the set $J$ is equal to $\{1,2, \ldots, n\})$ then the above definition coincides with the definition of joint left spectrum in [3]. We remark that $\left(\lambda_{j}\right)_{j \in J} \in \operatorname{Sp}(\mathbf{T})$ iff for any $N \in \mathbf{N}$ and $j_{1}, j_{2}, \ldots, j_{N} \in J$ it is the case that $\left(\lambda_{j_{1}}, \lambda_{j_{2}}, \ldots, \lambda_{j_{N}}\right) \in \operatorname{Sp}\left(T_{j_{1}}, T_{j_{2}}, \ldots, T_{j_{N}}\right)$. These remarks enable us to use the following result obtained by Patel (see [3], Proposition 1.2).

1.3. Proposition. Let $\lambda_{1}, \lambda_{2}, \ldots, \lambda_{N}$ be complex numbers and $T_{1}, T_{2}, \ldots, T_{N}$ be closed linear operators with a common domain $\mathscr{D}$. Then $\left(\lambda_{1}, \lambda_{2}, \ldots, \lambda_{N}\right) \in$ $\operatorname{Sp}\left(T_{1}, T_{2}, \ldots, T_{N}\right)$ if and only if there is a sequence $\left\{y_{k}\right\}_{k=1}^{\infty}$ of unit vectors from $\mathscr{D}$ such that $\lim _{k \rightarrow \infty}\left(T_{n} y_{k}-\lambda_{n} y_{k}\right)=0$, for every $n=1,2, \ldots, N$.

Remark. In Proposition 1.2 of [3] it is assumed that the domain $\mathscr{D}$ is dense in $H$. However, an examination of the proof shows that the result remains valid without this hypothesis. 
Let now $E$ be a spectral measure defined on a $\sigma$-algebra $\mathscr{B}$ of subsets of a topological space $\Omega$. Denote by $M(\Omega)$ the set of all equivalence classes of complex $\mathscr{B}$-measurable functions on $\Omega$ and by $L^{\infty}(\Omega)$ its subset formed by the $E$-essentially bounded functions.

1.4. Definition. Let $f_{1}, f_{2}, \ldots, f_{N}$ be elements from $M(\Omega)$. The spectrum $\operatorname{Sp}_{L^{\infty}(\Omega)}\left(f_{1}, f_{2}, \ldots, f_{N}\right)$ is defined as the set of all $N$-tuples $\left(\lambda_{1}, \lambda_{2}, \ldots, \lambda_{N}\right)$ of complex numbers such that there do not exist elements $v_{1}, v_{2}, \ldots, v_{N}$ from $L^{\infty}(\Omega)$ satisfying $\sum_{n=1}^{N} v_{n}(z)\left[f_{n}(z)-\lambda_{n}\right]=1$ E-almost everywhere.

To complete our set of definitions we recall that the closed support, $\operatorname{supp} E$, of the measure $E$ is, by definition, the intersection of all closed, measurable sets of full $E$-measure, that is,

$$
\operatorname{supp} E=\cap\{\sigma \in \mathscr{B}: \sigma \text { is closed and } E(\Omega \backslash \sigma)=0\} .
$$

\section{The main result}

Before stating the main theorem we will separate here a part of its proof as a separate lemma.

2.1. Lemma. Let $\mathbf{T}=\left(T_{j}\right)_{j \in J}$ be a doubly commuting family of normal, not necessarily bounded, operators in a Hilbert space $H$ such that $D\left(T_{i} T_{j}^{*} T_{j}\right)=$ $D\left(T_{i}\left(I+T_{j}^{*} T_{j}\right)\right)$ for all $i, j \in J$. Then the resolutions of the identity of the operators $T_{j}, j \in J$, commute.

Proof. In [2] $\$ 12$ it has been shown that the values of the spectral measure of an unbounded normal operator $T$ belong to the $W^{*}$-algebra generated by the coefficients of its characteristic matrix, that is, the operators: $\left(I+T^{*} T\right)^{-1}$, $T\left(I+T^{*} T\right)^{-1}, T^{*}\left(I+T T^{*}\right)^{-1}, T T^{*}\left(I+T T^{*}\right)^{-1}$, (the above algebra is commutative if $T$ is normal, see [4] Theorem 20). Therefore to prove the lemma it is sufficient to prove that all the coefficients of the characteristic matrices of $T_{j}$, $j \in J$, commute with one another, as then the algebra they generate will also be commutative and the values of the spectral measures of $T_{j}, j \in J$, are in this algebra.

Some of the required computations were done in [3]. As all of them can be shown similarily let us see, for example, how to establish the following equality: $T_{i}\left(I+T_{i}^{*} T_{i}\right)^{-1}\left(I+T_{j}^{*} T_{j}\right)^{-1}=\left(I+T_{j}^{*} T_{j}\right)^{-1} T_{i}\left(I+T_{i}^{*} T_{i}\right)^{-1}$. There is $T_{i} T_{j}^{*} T_{j}=$ $T_{j}^{*} T_{i} T_{j}=T_{j}^{*} T_{j} T_{i}$ as $T_{i}$ and $T_{j}$ doubly commute, and thus $T_{i}\left(I+T_{j}^{*} T_{j}\right)=$ $\left(I+T_{j}^{*} T_{j}\right) T_{i}$. Hence $\left(I+T_{j}^{*} T_{j}\right)^{-1} T_{i}\left(I+T_{j}^{*} T_{j}\right)=\left(I+T_{j}^{*} T_{j}\right)^{-1}\left(I+T_{j}^{*} T_{j}\right) T_{i} \subset$ $T_{i}$ and therefore $\left(I+T_{j}^{*} T_{j}\right)^{-1} T_{i} \subset T_{i}\left(I+T_{j}^{*} T_{j}\right)^{-1}$. As $\left(I+T_{j}^{*} T_{j}\right)^{-1}$ is bounded 
and $T_{i}$ is normal then also $\left(I+T_{j}^{*} T_{j}\right)^{-1} T_{i}^{*} \subset T_{i}^{*}\left(I+T_{j}^{*} T_{j}\right)^{-1}$. It follows that $\left(I+T_{j}^{*} T_{j}\right)^{-1} T_{i}^{*} T_{i} \subset\left(T_{i}^{*} T_{i}\right)\left(I+T_{j}^{*} T_{j}\right)^{-1}$ which in turn implies that $\left(I+T_{j}^{*} T_{j}\right)^{-1}\left(I+T_{i}^{*} T_{i}\right) \subset\left(I+T_{i}^{*} T_{i}\right)\left(I+T_{j}^{*} T_{j}\right)^{-1}$. Thus

$$
\left(I+T_{i}^{*} T_{i}\right)^{-1}\left(I+T_{j}^{*} T_{j}\right)^{-1} \subset\left(I+T_{j}^{*} T_{j}\right)^{-1}\left(I+T_{i}^{*} T_{i}\right)^{-1}
$$

and since the operators on both sides are bounded we have an equality here. Using this equality and the inclusion $\left(I+T_{j}^{*} T_{j}\right)^{-1} T_{i} \subset T_{i}\left(I+T_{j}^{*} T_{j}\right)^{-1}$, obtained earlier, we can reach our goal as follows:

$$
\begin{aligned}
\left(I+T_{j}^{*} T_{j}\right)^{-1} T_{i}\left(I+T_{i}^{*} T_{i}\right)^{-1} & \subset T_{i}\left(I+T_{j}^{*} T_{j}\right)^{-1}\left(I+T_{i}^{*} T_{i}\right)^{-1} \\
& =T_{i}\left(I+T_{i}^{*} T_{i}\right)^{-1}\left(I+T_{j}^{*} T_{j}\right)^{-1} .
\end{aligned}
$$

Noting that the left-hand side operator is bounded we can once more deduce that the inclusion is in fact an equality.

Before stating the main theorem we will need some further notation. Let $\mathbf{T}=\left(T_{j}\right)_{j \in J}$ be a doubly commuting family of normal operators. Let $\Omega=\mathbf{C}^{J}$, equipped with the product topology, and $\mathscr{B}$ be the $\sigma$-algebra generated by all the cylindric sets in $\Omega$, that is, sets of the form $C=\prod_{j \in J} C_{j}$ where each $C_{j}$ is a Borel subset of $\mathbf{C}$ and $C_{j}=\mathbf{C}$ for all but a finite collection of the indices $j$. Let $p_{i}$ denote the projection onto the $i$ th factor given by $p_{i}: \mathbf{C}^{J} \ni\left(z_{j}\right)_{j \in J} \rightarrow z_{i} \in \mathbf{C}$.

2.2. TheOREM. For any family $\mathbf{T}=\left(T_{j}\right)_{j \in J}$ of doubly commuting normal operators fulfilling the assumptions of Lemma 2.1 there exists a spectral measure $E$ on $(\Omega, \mathscr{B})$ such that $T_{j}=\int_{\Omega} p_{j} d E, j \in J$. For such a measure $E$, its closed support coincides with the joint spectrum of the given family, that is, $\operatorname{supp} E=\operatorname{Sp}(\mathbf{T})$.

PROOF. Lemma 2.1 together with the known method of constructing the joint spectral measure from a collection of commuting ones (see [1], \$3 or [2], Lemma 9) gives immediately the first assertion. It remains to prove the required characterization of the support of this joint spectral measure.

Fix now a point $\left(\lambda_{j}\right)_{j \in J}$ belonging to $\mathbf{C}^{J}$. It will not belong to $\operatorname{supp} E$ iff there is an open cylindric set, from the basis of neighbourhoods of $\left(\lambda_{j}\right)_{j \in J}$, that is of $E$-measure zero. In other words iff there exist $N \in \mathbf{N}, j_{1}, j_{2}, \ldots, j_{N} \in J$ and $\varepsilon>0$ such that $E\left[C\left(\varepsilon ; j_{1}, j_{2}, \ldots, j_{N}\right)\right]=0$ where the cylindric set $C\left(\varepsilon ; j_{1}, \ldots, j_{N}\right)$ is given by $\left\{\left(z_{j}\right)_{j \in J}: \max _{1 \leqslant n \leqslant N}\left|z_{j_{n}}-\lambda_{j_{n}}\right|<\varepsilon\right\}$. For the time being fix $N \in \mathbf{N}$ and $j_{1}, j_{2}, \ldots, j_{N} \in J$. Let $C_{\varepsilon}=C\left(\varepsilon ; j_{1}, j_{2}, \ldots, j_{N}\right)$ whenever $\varepsilon>0$. The following conditions are equivalent:

(i) $E\left(C_{\varepsilon}\right)=0$ for some $\varepsilon>0$,

(ii) $\left(\lambda_{j_{1}}, \lambda_{j_{2}}, \ldots, \lambda_{j_{N}}\right) \notin \operatorname{Sp}_{L^{\infty}(\Omega)}\left(p_{j_{1}}, p_{j_{2}}, \ldots, p_{j_{N}}\right)$,

(iii) $\left(\lambda_{j_{1}}, \lambda_{j_{2}}, \ldots, \lambda_{j_{N}}\right) \notin \operatorname{Sp}\left(T_{j_{1}}, T_{j_{2}}, \ldots, T_{j_{N}}\right)$. 
The proof of the equivalence of (i) and (ii) has in fact been done in [3], Proposition 1.9 and we will it omit here. (One only needs to change the scalar measure $\mu$ to the spectral measure $E$; both are used only to determine the measure zero sets.)

Now, consider the set $\mathscr{A}_{E}=\left\{\int f d E: f \in M(\Omega)\right\}$. In [2], Proposition 1 it has been shown that this set under the opertions of the strong product, strong sum and scalar product of operators (that is, the closures of the usual operations of sum or product) forms an algebra and that the map: $\Phi: M(\Omega) \ni f \rightarrow\left(\int f d E\right) \in$ $\mathscr{A}_{E}$ is an algebraic homomorphism.

We will use this homomorphism to prove the implication from (ii) to (iii). Let then (ii) be fulfilled and let $v_{1}, v_{2}, \ldots, v_{N} \in L^{\infty}(\Omega)$ be as in the definition of $\mathrm{Sp}_{L^{\infty}(\Omega)}$ that is $\sum_{n=1}^{N} v_{n}(z)\left[p_{j_{n}}(z)-\lambda_{j_{n}}\right]=1 E$-almost everywhere. Then $I=\Phi(1)$ $=\sum_{n=1}^{N} \Phi\left(v_{n}\right) \circ\left[\Phi\left(p_{j_{n}}\right)-\lambda_{j_{n}} I\right] \supset \sum_{n=1}^{N} \Phi\left(v_{n}\right)\left(T_{j_{n}}-\lambda_{j_{n}} I\right)$ the first product and sum being in the strong sense. (We used here the formula $\Phi\left(p_{j_{n}}\right)=\int p_{j_{n}} d E=T_{j_{n}}$ ). But, if $v_{n} \in L^{\infty}(\Omega), 1 \leqslant n \leqslant N$, then $\Phi\left(v_{n}\right)=\int v_{n} d E$ is bounded and thus $\left(\lambda_{j_{1}}, \lambda_{j_{2}}, \ldots, \lambda_{j_{N}}\right) \notin \operatorname{Sp}\left(T_{j_{1}}, T_{j_{2}}, \ldots, T_{j_{N}}\right)$.

There remains to prove the implication from (iii) to (i). This can be achieved using Proposition 1.3. Namely, assume that (iii) is fulfilled but (i) is not. Then $E\left(C_{\varepsilon}\right) \neq 0$ for every $\varepsilon>0$. We will take $\varepsilon$ equal to $1 / m$ where $m$ runs through the natural numbers.

Let $C_{m}=C\left(1 / m ; j_{1}, j_{2}, \ldots, j_{N}\right)$ and let $x_{m}$ be a unit vector from $H$ such that $E\left(C_{m}\right) x_{m} \neq 0$. Furthermore, let $\chi_{m}$ be the characteristic function of the set $C_{m}$ and $\mu_{m}$ be the scalar measure connected with $x_{m}$, that is, $\mu_{m}(\sigma)=\left\langle E(\sigma) x_{m}, x_{m}\right\rangle$. Put $s_{m}=\left[\mu_{m}\left(C_{m}\right)\right]^{1 / 2}, f_{m}=s_{m}^{-1} \chi_{m}$ and $y_{m}=\left(\int f_{m} d E\right) x_{m}$.

The functions $f_{m}$ are from $L^{\infty}(\Omega)$ and so the $y_{m}$ are certainly well defined since each $x_{m} \in D\left(\int f_{m} d E\right)=H$. We will show that the sequence $\left\{y_{m}\right\}_{m=1}^{\infty}$ fulfills the requirements of Proposition 1.3. Indeed, their norm is equal to 1 since

$$
\left\|y_{m}\right\|^{2}=\int\left|f_{m}\right|^{2} d \mu_{m}=\left(1 / s_{m}^{2}\right) \int_{C_{m}} 1 d \mu_{m}=1 .
$$

Now $\Phi\left(f_{m}\right)$ are bounded operators so the strong product and product coincide in the equation

$$
\Phi\left[\left(p_{j_{n}}-\lambda_{j_{n}}\right) f_{m}\right]=\Phi\left(p_{j_{n}}-\lambda_{j_{n}}\right) \Phi\left(f_{m}\right)=\left(T_{j_{n}}-\lambda_{j_{n}} I\right) \Phi\left(f_{m}\right) .
$$

Hence to show that $y_{m}, m=1,2, \ldots$, are in the domain of each $\left(T_{j_{n}}-\lambda_{j_{n}} I\right)$, $1 \leqslant n \leqslant N$, and to compute the norms of their images it is enough to show that the vectors $x_{m}$ are in the domains of the operators $\int\left(p_{j_{n}}-\lambda_{j_{n}}\right) f_{m} d E$ and to compute the norms of their images by these operators. But,

$$
\int\left|p_{j_{n}}-\lambda_{j_{n}}\right|^{2}\left|f_{m}\right|^{2} d \mu_{m}=\left(s_{m}\right)^{-2} \int_{C_{m}}\left|p_{j_{n}}-\lambda_{j_{n}}\right|^{2} d \mu_{m} \leqslant 1 / m^{2}<\infty
$$


as in the set $C_{m}$ we have $\left|\left(p_{j_{n}}-\lambda_{j_{n}}\right)(z)\right| \leqslant 1 / m, z \in C_{m}$. Thus the $y_{m}$ are indeed in the domain of $\left(T_{j_{n}}-\lambda_{j_{n}} I\right), 1 \leqslant n \leqslant N$, and what is more $\left\|T_{j_{n}} y_{m}-\lambda_{j_{n}} y_{m}\right\|^{2} \leqslant$ $1 / m^{2} \rightarrow 0$ as $m \rightarrow \infty$.

Now Proposition 1.3 gives the contradiction $\left(\lambda_{j_{1}}, \lambda_{j_{2}}, \ldots, \lambda_{j_{N}}\right) \in$ $\operatorname{Sp}\left(T_{j_{1}}, T_{j_{2}}, \ldots, T_{j_{N}}\right)$. This ends the proof of the equivalence of our three conditions and the entire proof as well. For, if (iii) is true for certain $N \in \mathbf{N}$ and $j_{1}, j_{2}, \ldots, j_{N} \in J$, then $\left(\lambda_{j}\right)_{j \in J} \notin \mathrm{Sp} T$. But, we have already noticed that if (i) is true for some $\varepsilon>0, N \in \mathbf{N}$ and $j_{1}, j_{2}, \ldots, j_{N} \in J$ then $\left(\lambda_{j}\right)_{j \in J} \notin \operatorname{supp} E$.

\section{Remarks}

It should be mentioned that in general the support of the measure $E$ need not be $\mathscr{B}$-measurable [1], $\$ 6$ and so the integral in Theorem 2.2 cannot be taken over $\mathrm{Sp}(\mathbf{T})$ as in the paper [3] of A. B. Patel. In the case of a finite family the essential range that Patel uses in his construction, that is, the set $\mathscr{E}\left(u_{1}, u_{2}, \ldots, u_{N}\right)$ by definition equal to

$\left\{\left(\lambda_{1}, \lambda_{2}, \ldots, \lambda_{N}\right) \in \mathbf{C}^{N}: \mu\left\{z \in \Omega: \sum_{n=1}^{N}\left|u_{n}(z)-\lambda_{n}\right|<\varepsilon\right\} \neq 0\right.$ for every $\left.\varepsilon>0\right\}$

determines the values of $\left(u_{1}, u_{2}, \ldots, u_{N}\right)$ up to a set of measure zero. One could try to define an essential range of a family $\left(u_{j}\right)_{j \in J}$ in the same way that has been done for the joint spectrum in Definition 1.2 in which case the equality $\operatorname{Sp}(\mathbf{T})=$ $\mathscr{E}\left[\left(p_{j}\right)_{j \in J}\right]$ will hold. But, in the case when the set $J$ is uncountable one cannot conveniently extend a function $f$, defined on $\operatorname{Sp}(\mathbf{T})$, in such a way that $f \circ u$, where $u=\left(p_{j}\right)_{j \in J}$, is a $\mathscr{B}$-measurable function on the whole of $\mathbf{C}^{J}$. This is an essential obstruction in Patel's method of constructing the joint spectral measure of the family.

It is no accident that this is so. There are families of non-trivial normal operators for which $\operatorname{supp} E$ (or equivalently $\operatorname{Sp}(\mathrm{T})$ ) is void. An example is discussed in [1], $\$ 7$.

It is always the case that $\operatorname{Sp}(\mathrm{T}) \subset \prod_{j \in J} \sigma\left(T_{j}\right)$. We have just mentioned that one extreme case of this inclusion is the possibility that $\operatorname{Sp}(\mathbf{T})=\varnothing$. It can also happen that $\operatorname{Sp}(\mathbf{T})=\mathbf{C}^{J}$. Of course, one can take the family $\mathbf{T}$ consisting of a single operator $T$ with its spectrum equals to $\mathbf{C}$. To provide an example for the case when the family of operators is more numerous we cannot simply take each $T_{j}$ equal to the chosen operator $T$. One can easily prove that in such a case the joint spectrum is a subset of the diagonal of $\mathbf{C}^{J}$; actually it is equal to

$$
\left\{\left(\lambda_{j}\right)_{j \in J} \in \mathbf{C}^{J}: \forall_{j \in J} \lambda_{j}=\mu \text {, for certain } \mu \in \boldsymbol{\sigma}(T)\right\} .
$$


So, to construct a more general example take the Hilbert space $H$ equal to $l^{2}\left(\mathbf{C}^{\mathbf{R}}\right)$ and let $F$ be the spectral measure defined on all subsets of $\mathbf{C}^{\mathbf{R}}$ by $F(\sigma) H=l^{2}(\sigma)=\{x \in H$ : supp $x \subset \sigma\}$, for each $\sigma \subset \mathbf{C}^{\mathbf{R}}$. As the set of indices $J$ we take R. Let $g_{j}: \mathbf{C}^{\mathbf{R}} \ni f \rightarrow f(j), j \in \mathbf{R}$ and $T_{j}=\int g_{j} d F$. Then all $T_{j}$ are normal and their resolution of the identity are given by $P_{j}(\tau)=F\left[g_{j}^{-1}(\tau)\right]$ and hence commute.

Take now any $\left(\lambda_{j}\right)_{j \in J} \in \mathbf{C}^{J}=\mathbf{C}^{\mathbf{R}}$. We will show that it belongs to $\mathrm{Sp}(\mathbf{T})$ by finding a point $f \in \mathbf{C}^{\mathbf{R}}$ such that $\left(T_{j}-\lambda_{j} I\right)$ is zero on $F(\{f\}) H$ for each index $j \in J$. As $F(\{f\}) \neq 0$ this will mean that for any bounded operators $B_{1}, B_{2}, \ldots, B_{N}$ and any indices $j_{1}, j_{2}, \ldots, j_{N} \in J$ the operator $\sum_{n=1}^{N} B_{n}\left(T_{j_{n}}-\lambda_{j_{n}}\right) I$ is zero on the space $F(\{f\}) H$ and thus cannot be included into the identity $I$. This means that $\left(\lambda_{j}\right)_{j \in J}$ lies in $\mathrm{Sp}(\mathrm{T})$.

For the required point it is enough to take $f: J=\mathbf{R} \ni j \rightarrow \lambda_{j} \in \mathbf{C}$, where $\left(\lambda_{j}\right)_{j \in J} \in \mathbf{C}^{\mathbf{R}}$ is the fixed arbitrary point of $\mathbf{C}^{J}$. Indeed, it then follows that $\left(T_{j}-\lambda_{j} I\right) F(\{f\})=\int_{\{f\}}\left(g_{j}-\lambda_{j}\right) d F=\left(g_{j}(f)-\lambda_{j}\right) F(\{f\})=(f(j)-$ $\left.\lambda_{j}\right) F(\{f\})=0$. Thus the constructed family $T$ has the desired property that its joint spectrum fills the whole $\operatorname{space} \operatorname{Sp}(\mathbf{T})=\mathbf{C}^{J}$.

The above examples illustrate the usual deficiency of measure theory when applied to more then a countable number of objects. The connection between the given family $T$ and the support of its joint spectral measure can then become more vague and unspecified and the classical results more difficult to generalize.

The author is grateful to the referee for helpful comments.

\section{References}

[1] Ju. M. Berezanskiir, 'Projective spectral theorem', Uspehi Mat. Nauk 39 (1984), 3-52, (in Russian); Russian Math. Surveys, 39:4 (1984), 1-62.

[2] J. Niechwiej, 'On commutative algebras of unbounded operators', Studia Math. 78 (1984), 217-230.

[3] A. B. Patel, 'A joint spectral theorem for unbounded normal operators, J. Austral. Math. Soc. 34 (1983), 203-213.

[4] M. H. Stone, 'On unbounded operators in Hilbert space', J. Indian Math. Soc. 15 (1951), 155-192.

Instytut Matematyki

Uniwersytet Jagielloński

ul. Reymonta 4

30-059 Kraków

Poland 